\title{
BIOINSPIRED PsI INTELLIGENT CONTROL FOR AUTONOMOUS DYNAMIC SYSTEMS
}

\author{
Farbod Khoshnoud, ${ }^{*}$ Clarence W. de Silva, ${ }^{* *}$ and Ibrahim I. Esat*
}

\begin{abstract}
Psi Intelligent Control aims to provide a framework for controlling autonomous dynamic systems with prediction capabilities inspired by Psi precognition. Psi in nature relates to an event or state not yet experienced. Although Psi phenomenon is unexplained, it is an inspiration for the present study. The research intends to define and develop frameworks and systems capable of providing information for predicting future events (e.g., for motion control of an autonomous vehicle) and use that information in control of autonomous dynamic systems. A generalized approach inspired by Psi precognition is proposed, and the effect of this technique in the response of dynamic systems is explored. The corresponding parameters and constraints to develop and analyze Psi Intelligent Control for dynamic systems are discussed. Obtaining optimized solutions while considering uncertainties in the system and input parameters are investigated. Optimal Uncertainty Quantification is used to obtain optimized solutions for the control of the autonomous dynamic system, with imperfectly known response functions, input probability measures and parameters.
\end{abstract}

\section{Key Words}

Bio-inspired systems, Psi precognition, control of dynamic systems, multi-agent robots, swarm robotics, connected vehicles

\section{Introduction}

The term Psi denotes anomalous processes of information or energy transfer that are currently unexplained in terms of known physical or biological mechanisms [1]. Although Psi phenomenon is unexplained, it is an inspiration for the present study. The representation of the prediction of future events for control of dynamic systems is explained in a framework inspired by Psi precognition. An introduction to this research was presented by the authors in

* Department of Mechanical Engineering, Brunel University London, Uxbridge, Middlesex UB8 3PH, United Kingdom; e-mail: \{Farbod.Khoshnoud, Ibrahim.Esat\}@brunel.ac.uk

** Department of Mechanical Engineering, University of British Columbia, Vancouver, V6T 1Z4, Canada; e-mail: desilva@ mech.ubc.ca

Recommended by Prof. Maoqing Li

(DOI: 10.2316/Journal.201.2015.4.201-2740) reference [2]. The current paper intends to extend the investigation and generalization of the framework of the Psi Intelligent Control in the context of Optimal Uncertainty Quantification (OUQ) [3] and Machine Wald [4]. Model predictive control (MPC) strategies rely on the model of the dynamic system and also depend on the physical limitations of sensors and actuators response times. A limitation of MPC can be considered as the running time for the online optimization algorithm which can potentially require substantial time and computational resources [5]. Psi Intelligent Control uses an agent ahead of time of the dynamic system (e.g., traveling ahead of a moving vehicle in the intended trajectory path of the vehicle) to provide information about the future (e.g., obstacles ahead which are not detected by the vehicle sensor systems due to sensor limitations) for the dynamic system (e.g., vehicle) under investigation.

Examples of various systems that use multi-agent collaborative schemes for control can be referred to as swarm robotics and multi-agent/robot systems, connected vehicles $(\mathrm{CVs})$, etc., where multiple entities present in the system and communicate with each other to perform a task. In swarm robotics population, decentralized and autonomous control, homogeneity, scalability, and functional extension are distinguished factors compared with multiagent and multi-robot systems [6]. Obstacle avoidance is a basic task in swarm robotics and multi-robot operations. Various approaches are used for obstacle avoidance such as potential fields [7], vision-based control frameworks (e.g., [8]), tracking controllers for motion coordination of multiple mobile robots [9], formation tracking control of unicycle-type mobile robots [10], atomic nucleus inspired algorithm for guiding robots around obstacles [11], formation transition based on geometrical features for multiple autonomous mobile robots [12], and particle swarm optimization techniques [13].

Another relevant problem is the CV technology in the development of self-driving vehicles, auto highways, auto intersection managements, and so on. According to the Research and Innovative Technology Administration (RITA) of the US Department of Transportation (DOT), the CV technology can potentially improve transportation by reducing $81 \%$ of all vehicle target crashes, $83 \%$ of all 
light vehicle target crashes, and $72 \%$ of all heavy truck target crashes annually [14]. It will also improve the congestion problem in the United States, which consumes up to 4.2 billion hours of traffic and 2.8 billion gallons of fuel annually. Some recent advances in the CV technology have been addressed here as follows. A linear programming formulation for autonomous intersection control within a dynamic traffic assignment under the $\mathrm{CV}$ environment has been developed to solve autonomous intersection control [15]. Using CV technology to improve the efficiency of intersections can result in up to $7 \%$ decrease in delay [16].

Traffic signal control strategies mainly rely on an infrastructure-based vehicle detector. They are generally point detectors, which cannot directly provide measurement of vehicle location and speed. A real-time adaptive signal phase allocation algorithm using $\mathrm{CV}$ data can be used for signal control by optimizing the phase sequence and duration with the two objective functions: minimization of total vehicle delay and minimization of queue length. Such control algorithm can reduce total delay by up to $16.33 \%$ in high and low penetration rate cases [17].

Acceleration-based connected cruise control (CCC), where CCC uses acceleration signals received from multiple vehicles ahead through wireless vehicle-to-vehicle communication, can increase roadway traffic mobility [18]. Realtime driver's compliance to the posted speed limit has been used to adjust the vehicle optimal speed limit values, which shows up to $20 \%$ of total travel time reductions, 6\%-11\% of safety improvements, and 5\%-16\% reduction in fuel consumptions [19].

The mathematical model of the behavior of a human driver, who repetitively learns the correct action from the past experience, can improve stability and the effectiveness for path-following of autonomous vehicles [20].

A CCC strategy that controls the velocity of multiple preceding cars, which changes with memory, is designed to improve roadway traffic, enhance safety, and reduce fuel consumptions and exhaust emissions. The CCC strategy receives signals of velocity changes from multiple cars ahead through wireless vehicle-to-vehicle communication and the relative distance and velocity difference of the immediately ahead car, using radar [21].

The technology proposed in this paper focuses on motion control (e.g., trajectory planning) and obstacle avoidance in two- and three-dimensional cases correspond to aerial, ground, or underwater vehicles. In case of aerial vehicles, Unmanned Aerial Vehicles (UAVs) are increasingly used for civilian and military applications including search and rescue, border interdiction, traffic monitoring, law enforcement, disaster and emergency management, wild fire suppression, communications relay, intelligence, reconnaissance, surveillance missions, and so on [22]. For aerial vehicle navigation, data including position, velocity, and attitude are used for guidance and control. Inertial navigation sensing systems are used for angular velocity and acceleration, and external non-inertial navigation data [e.g., by a satellite-based system such as the Global Positioning System (GPS)] for inevitable drift. GPS is not always available, and therefore visual (imagebased) navigation is used as an additional sensing system.
Landmark-based approaches use on-board cameras to take images of selected landmarks during flight and by matching these images with referenced data which can estimate the position and attitude of the air vehicle relative to the landmark [23], [24]. An algorithm that takes into account kinematic and dynamic properties, and also the navigational capabilities of the air vehicle, can determine a shortest trajectory of a fixed-wing UAV [25]. This technique can cope with long-term GPS outages. The algorithm is based on a discretization of the airspace by a specific network. In motion planning, it should be noted that, the dynamic constraints do not allow air vehicles to instantaneously change their velocity or perform sharp turns (also known as kinodynamic problems) [26]. Different techniques have been used in finding a collision-free path of a robot in an environment with obstacles including mixed integer linear programs, the potential field method, cell decomposition, the roadmap method, the mass-spring-damper method, and several network-based approaches [27], [28].

In dealing with flight path problems, a simplified flight performance model is considered in various approaches such as the network-based methods [29]-[31] that generate a polygonal path from a regular grid discretization of the airspace. Other methods include performing a path smoothing after obtaining a collision-free path to determine a shortest path in a network based on Voronoi polygons [32] which achieves smoothing of the path using a spring-mass approach, or using a series of cubic splines to smooth the straight-line segments [33]. Solving the flight path problem that accounts for no-fly zones and differential constraints can use sampling-based methods, which imply rapidly exploring random trees, MPC methods, and mathematical programming methods based on route planning optimization [34]. Optimization approaches are also used for multiple heterogeneous UAVs, with cooperative decision making and control, which include vehicles with different operational capabilities and kinematic constraints [35]. Bioinspired designs are used for autonomous flight formation of multiple flapping-wing flying vehicles using motion capture system [36].

\section{Psi Intelligent Control}

The concept of Psi Intelligent Control is represented schematically by the example in Figs. 1 and 2 . In the example in Fig. 1, a vehicle is traveling with the speed of $\nu$ and is required to avoid obstacles. The vehicle's sens-

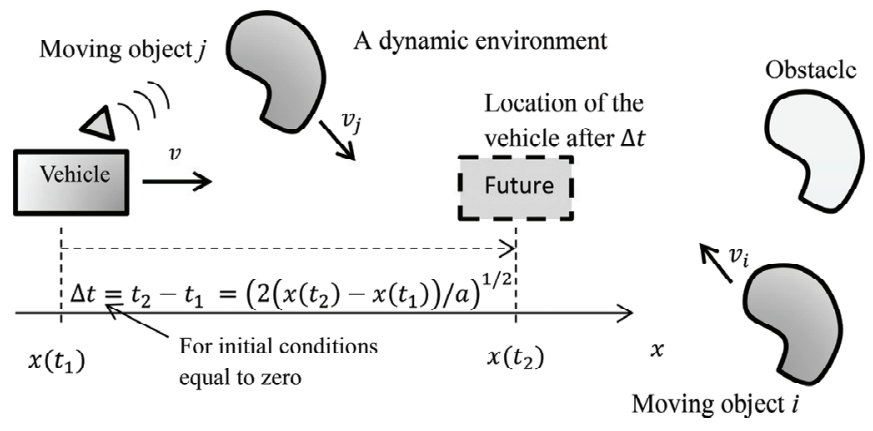

Figure 1. A vehicle in a dynamic environment. 
ing range is limited. An agent is traveling ahead of time in the "future" location (Figs. 1 and 2) of the vehicle in the intended trajectory path of the vehicle [2]. The agent sensors can detect the obstacles in the "future" location of the vehicle and inform the vehicle to avoid obstacles and plan its trajectory ahead of time (before the vehicle itself can detect) controllers. This allows the control system to adjust actuators with the sensors information that "predicting/feeling the future" of the vehicle with $\Delta t$ time before the vehicle can detect the events/obstacles.

In existing multi-robot/agent, swarm, CVs, etc., technologies, each entity (e.g., robot, agent, vehicles) is considered as a system equipped with sensors used in the corresponding control systems for performing various tasks. The sensing in such technologies is limited to the

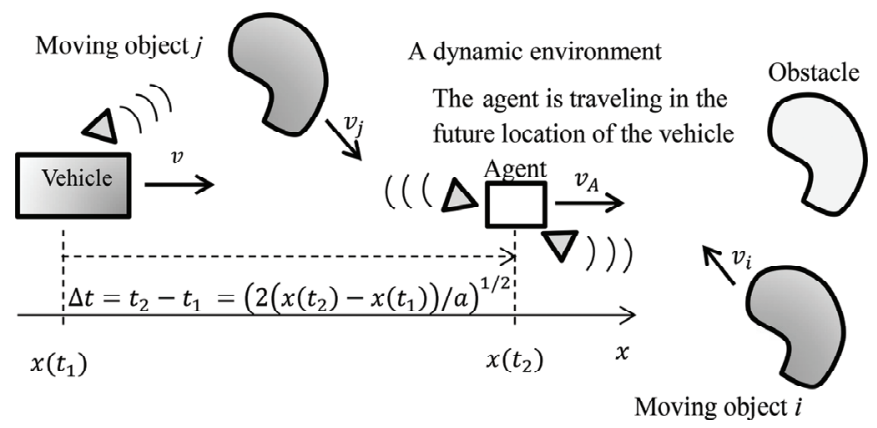

Figure 2. An agent is traveling in the future location of the vehicle. time and location of the system. The Psi Control concept explores the possibility of eliminating sensing limitations by predicting the future, where the sensing system is an agent dedicated to provide necessary information not limited to location of the vehicle/robot under consideration. The Psi Intelligent Control concept is a challenge to fulfill the requirements of knowing the future status of the vehicle with no limitation.

A feedback control strategy is represented as in Fig. 3. The controller is considered as a switch $\mathrm{C}_{\mathrm{d}}(\mathrm{s})$ which decides to apply the current sensor information, or the "future" information. $\mathrm{C}_{1}(\mathrm{~s})$ is a conventional obstacle avoidance algorithm where the Sensor V (Fig. 3) detects the objects and the actuator drives the vehicle around the obstacle using a feedback control algorithm. The controller $\mathrm{C}_{2}(\mathrm{~s})$ receives information from the agent Sensor $\mathrm{A}$ and therefore allows $\Delta t$ more time for control decision making and adjustment due to the sensor information from Sensor A which is $\Delta t$ ahead of time. The switching control scheme can be defined by: If Sensor $V$ is detecting obstacle(s) then Switch to Controller $C_{1}$ Else Switch to Controller $\mathrm{C}_{2}$ End.

Figure 4 illustrates an example where a vehicle is traveling from $\mathrm{A}$ to $\mathrm{F}$ and the aim is to avoid obstacles in a minimum time travel. The agent provides information about the obstacles in the trajectory path of the vehicle and therefore decides the shortest distance path $\mathrm{AB}_{1} \mathrm{C}$. It continues the travel by knowing the obstacles in the trajectory path (by the information received from agent

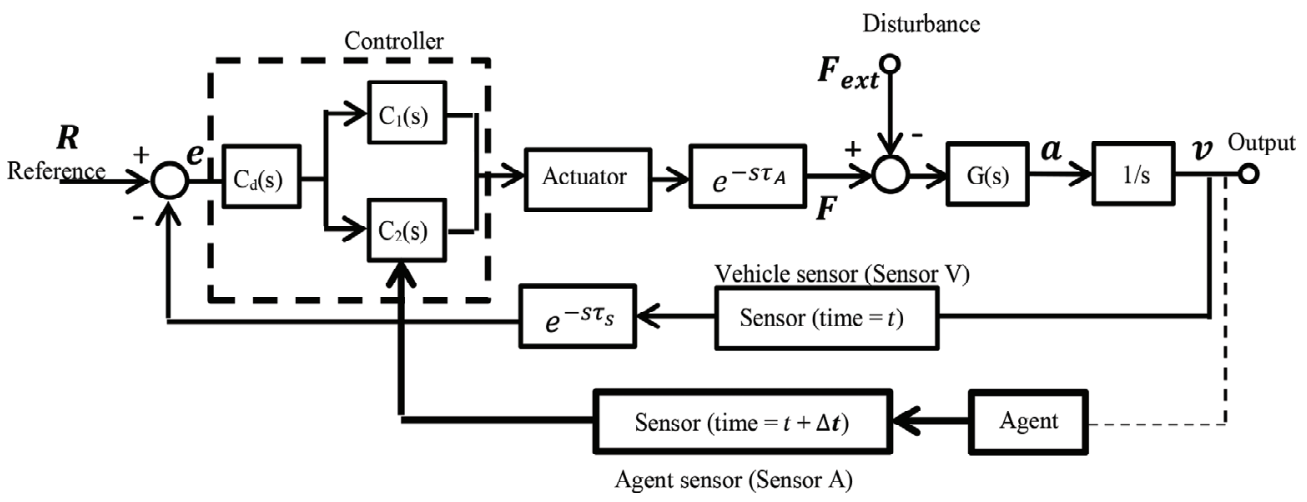

Figure 3. A block diagram for Psi Intelligent Control.

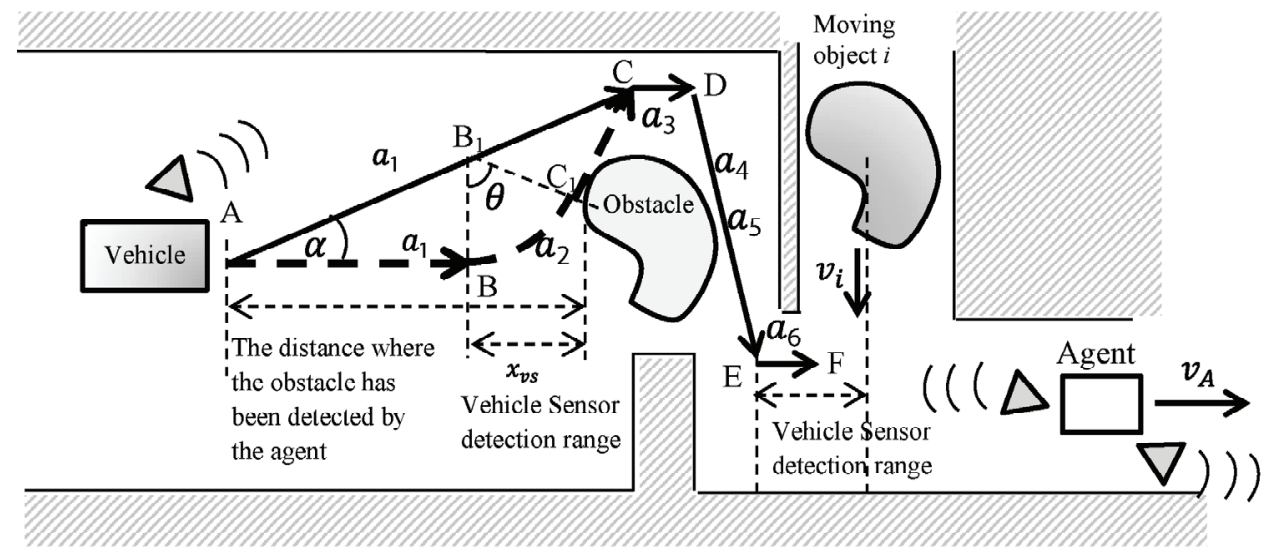

Figure 4. An example for Psi Intelligent Control. 
sensor), and stops at E to avoid collision. Now consider the case when the information from the agent sensor is not available. The vehicle chooses the path $\mathrm{AB}$ until the vehicle sensor can detect the obstacle. It can be shown that the overall travel time for each section can be minimized when sensor information is received from the agent by applying the maximum acceleration by the vehicle's propulsion system [2]. In addition to the potential for improving the overall traveling time, the vehicle control with no agent sensor can fail in avoiding collision at location $\mathrm{F}$ (if the vehicle sensor is not able to detect the moving object).

The control parameters can be designed for minimizing traveling time or energy depending on the application requirements.

This research intends to define and develop frameworks and systems capable of providing information for predicting future events (e.g., for motion control of an autonomous vehicle) and use that information in control of autonomous dynamic systems. For analyzing a dynamic system and developing a robust control strategy, it is required to consider uncertainties in the system and deal with imperfectly known information. Therefore, it is desired to obtain optimized solutions while considering uncertainties in the dynamic system and input parameters. OUQ [3] is used here as a general framework to obtain optimized solutions for Psi Intelligent Control applied to dynamic systems, which considers imperfectly known response functions, input probability measures, and parameters. The uncertain information can include geometry, obstacles, velocity, acceleration, the controller system parameters (e.g., time delays, and sensor and actuator limitations), and disturbances to the system (e.g., wind gust to a UAV). The general representation of the problem can be formulated as follows.

For function $G: \mathcal{X} \rightarrow \mathbb{R}, X \rightarrow G(X)$, and probability of $\mathbb{P} \in \mathcal{M}(\mathcal{X})$, we want to certify that the probability of function $G(X)$ to be greater than $b$ (i.e., to fail) is less than $\epsilon$, which can be written as

$$
\mathbb{P}[G(X) \geq b] \leq \epsilon
$$

We know $(G, \mathbb{P}) \in \mathcal{A}$, and the admissible extremal scenarios $\mathcal{A}$ is $[3]$

$$
\mathcal{A} \subset\left\{(g, \mu) \mid \begin{array}{l}
g: \mathcal{X} \rightarrow \mathbb{R} \\
\mu \in \mathcal{P}(\mathcal{X})
\end{array}\right\} .
$$

For $n$ number of inputs, $\mathcal{X}_{i}, i=1, \ldots, n$, and a bounded mean output, $\mathbb{E}_{\mu}[g], \mathcal{A}$ is defined as

$$
\mathcal{A}:=\left\{(g, \mu) \mid \begin{array}{c}
g: \mathcal{X}_{1} \times \cdots \times \mathcal{X}_{m} \rightarrow \mathbb{R} \\
\mu=\mu_{1} \otimes \mu_{2} \otimes \cdots \otimes \mu_{m} \\
m_{1} \leq \mathbb{E}_{\mu}[g] \leq m_{2}
\end{array}\right\}
$$

where $\mu_{i}$ is the probability measure of the input parameter $\mathcal{X}_{i}\left(\mu_{i} \in \mathcal{P}\left(\mathcal{X}_{i}\right)\right)$ and $g$ is a possible transfer function of the system $(G)$, which gives the output value for input/parameter $\mathcal{X}_{i}$. The original problem entails optimizing over a collection of $(g, \mu)$ that could be $(G, \mathbb{P}) . \mathbb{E}_{\mu}$ denotes the average output value (e.g., time or energy) for the corresponding uncertain parameters, $\mu ; m_{1}$ and $m_{2}$ are output lower and upper bounds, respectively, if known; and $\mu_{i}$ parameters are constrained values with corresponding lower and upper bounds for each parameter.

The optimal bounds on the probability of the system input can be described by the upper bound given by

$$
\mathcal{U}(\mathcal{A}):=\sup _{(f, \mu) \in \mathcal{A}} \mu[g(X) \geq b]
$$

and for the lower bound as

$$
\mathcal{L}(\mathcal{A}):=\inf _{(f, \mu) \in \mathcal{A}} \mu[g(X) \geq b]
$$

where the optimal bounds on the probability of the controller output for providing a required performance can be expressed as

$$
\mathcal{L}(\mathcal{A}) \leq \mathbb{P}[G(X) \geq b] \leq \mathcal{U}(\mathcal{A})
$$

The output lower and upper bounds (range) are therefore obtained by solving a constrained optimization problem, $\mathcal{U}(\mathcal{A})$, with the inputs/parameters being constrained as $[3]$

$$
\mathcal{U}(\mathcal{A}):=\sup _{(G, \mu) \in \mathcal{A}} \mu[G(X) \leq 0]
$$

Solving the optimization problem can be carried out using any available technique (e.g., genetic algorithm).

If $\mathcal{U}(\mathcal{A}) \leq \epsilon$, then the system response is safe even in the worst case; if $\epsilon<\mathcal{L}(\mathcal{A})$, then the system is unsafe even in best case; and if $\mathcal{L}(\mathcal{A}) \leq \epsilon \leq \mathcal{U}(\mathcal{A})$, then more information about the system and parameters is required for analysis of the response of the system.

In a vehicle motion control problem, the propulsion force and moment vectors, $\boldsymbol{F}$ and $\boldsymbol{M}$, can be considered as the input to the system, and the displacement, $\boldsymbol{r}$, as the output. The corresponding acceleration and velocity vectors generated by the propulsion forcing vectors are bounded due to the physical limits and time delays. In such problem, the bounded parameters which include the physical limits of the vehicle and control systems, and geometrical constraints are as follows. The bounded input limits can be expressed as $\boldsymbol{F}(x, y, z) \in \mathcal{X}_{i}:=\left[\boldsymbol{F}_{\text {min }}, \boldsymbol{F}_{\text {max }}\right]$. For the corresponding acceleration and velocity vectors, the limits are defined as $\boldsymbol{a}_{\min } \leq \boldsymbol{a}_{1} \leq \boldsymbol{a}_{\max }$, and $\boldsymbol{v}_{\min } \leq \boldsymbol{v} \leq \boldsymbol{v}_{\max }$, respectively. The limits for the output due to the path geometrical constraints can be considered as $\boldsymbol{r}_{j} \in\left[\boldsymbol{r}_{j \_ \text {min }}, \boldsymbol{r}_{j}\right.$ max $]$, where vector $\boldsymbol{r}_{j}$ represents the geometry components (e.g., $x_{j}, y_{j}, z_{j}$ components of $\boldsymbol{r}_{j}$ ) with the corresponding limits $x_{j_{-} \min } \leq x_{j} \leq x_{j \_\max }, \quad y_{j_{-} \min } \leq y_{j} \leq y_{j \_\max }$, and $z_{j \_\min } \leq z_{j} \leq z_{j \_\max }$. The geometric constraint can also be defined as a function $D_{j}\left(x_{j}, y_{j}, z_{j}\right)$ for a threedimensional trajectory problem, where $j$ denotes the discretization of the geometry for each section $j(e . g$. , sections ABCDEF in Fig. 4).

A simplified example for motion control problem is given below. It is assumed that the control is performed in 
a single step (not as a feedback system) and the equation of motion, which includes the vehicle propulsion force and the external force excitations, is given by $\sum \boldsymbol{F}=m \frac{d^{2} \boldsymbol{r}(x, y, z, t)}{d t^{2}}$. In this problem, it is assumed that the acceleration and velocity bounds take into account the sensor and actuator limits and delays of the control system and the plant. Therefore, $\mathcal{A}$ can be given by

$$
\mathcal{A}:=\left\{(g, \mu) \mid \begin{array}{c}
g: \mathcal{X}_{1} \times \mathcal{X}_{2} \times \mathcal{X}_{3} \rightarrow \mathbb{R} \\
\mu=\mu_{1} \otimes \mu_{2} \otimes \mu_{3} \\
\boldsymbol{r}_{j_{-} \min } \leq \mathbb{E}_{\mu}\left[\boldsymbol{r}_{j}\right] \leq \boldsymbol{r}_{j \_\max } \\
g=\boldsymbol{r}_{j}
\end{array}\right\} .
$$

As an example, the geometric constraint can be considered as the geometry boundaries and obstacles in Fig. 4. Then the corresponding problem for optimizing time (or energy) becomes

$$
\mathcal{U}(\mathcal{A}):=\sup _{(r, \mu) \in \mathcal{A}} \mu\left[\boldsymbol{r}_{i}(X) \leq 0\right]
$$

Note that for optimizing energy the cost function $\boldsymbol{F} \cdot \boldsymbol{r}$ can be used as the output objective function. Therefore, in this framework if AC trajectory (in Fig. 4) is used, as for the vehicle with Psi control incorporated in, the optimized solution (when initial conditions are zero) is $t_{1}=\left(2 \overline{\mathrm{AB}_{1} \mathrm{C}} /\left(\frac{d^{2} r}{d t^{2}}\right)\right)^{1 / 2}$ for time optimization, where the $\mathrm{AB}_{1} \mathrm{C}$ trajectory is the solution of the optimization problem above. For the vehicle with no Psi control in place, the trajectory will lead to $\mathrm{ABC}$ trajectory. This is the same solution as before. However, the OUQ framework is applied here as a general solution of all problems in vehicle motion control, which can include any scenario not limited to the simplified example presented here. It should be noted that other parameters and bounds can be added to the OUQ including the actuator and sensor physical limits and time delays, and control parameters such as rise time, overshoot, and settling time.

The generalization of OUQ to sample data is equivalent to performing Bayesian Inference but optimizing the prior (i.e., taking the worst prior). This is also referred to as Machine Wald [4], where, if an estimation of a function $\boldsymbol{\Phi}(\mu)$, is function $\theta$ of sample data $d$, then the estimation error $\theta(d)-\boldsymbol{\Phi}(\mu)$ is required to be made as close as possible to zero.

\section{Conclusion}

A generalized approach inspired by Psi precognition was proposed in this paper and the effect of this technique in the response of dynamic systems was discussed. The corresponding parameters and constraints to develop and analyze Psi Intelligent Control for dynamic systems were addressed. Obtaining optimized solutions while considering uncertainties in the system and input parameters were investigated using OUQ for the control of the autonomous dynamic system, with imperfectly known response functions, input probability measures and parameters.

\section{Acknowledgement}

The first author would like to acknowledge Brunel Research and Innovation Fund Award for support of the research carried out in this paper.

\section{References}

[1] D.J. Bem, Feeling the future: Experimental evidence for anomalous retroactive influences on cognition and affect, Journal of Personality and Social Psychology, American Psychological Association, 100, 2011, 407-425.

[2] F. Khoshnoud, I.I. Esat, and C.W. de Silva, Bioinspired Psi intelligent control for autonomous vehicles, IEEE proceedings of the International Conference on Computer Science 8 Education, Cambridge, UK, 2015, 208-212.

[3] H. Owhadi, C. Scovel, T. Sullivan, M. McKerns, and M. Ortiz, Optimal uncertainty quantification, SIAM Review, 55(2), 2013, 271-345.

[4] H. Owhadi and C. Scovel, Towards Machine Wald, (Submitted on 10 Aug 2015, last revised 1 Oct 2015 (this version, v2)) http://arxiv.org/abs/1508.02449

[5] F. Borrelli, A. Bemporad, and M. Morari, Predictive control for linear and hybrid systems, Lecture notes, University of California, Berkeley, CA, 2014.

[6] Y. Tan and Z.-y. Zheng, Research advance in swarm robotics, Defense Technology, 9, 2013, 18-39.

[7] O. Khatib, Real-time obstacle avoidance for manipulators and mobile robots, International Journal of Robotics Research, 5(1), 1986, 500-505.

[8] A.K. Das, R. Fierro, V. Kumar, J.P. Ostrowski, J. Spletzer, and C.J. Taylor, A vision-based formation control framework, IEEE Transactions on Robotics and Automation, 18(5), 2002, 813-825.

[9] J.Y. Shao, G.M. Xie, J.Z. Yu, and L. Wang, A tracking controller for motion coordination of multiple mobile robots, IEEE/RSJ International Conference on Intelligent Robots and Systems, 2005, 783-788.

[10] K.D. Do, Formation tracking control of unicycle-type mobile robots with limited sensing ranges, IEEE Transactions on Control Systems Technology, 16(3), 2008, 527-538.

[11] E.M. Fikret, X. Li, and X.M. Liang, A regular tetrahedron formation strategy for swarm robots in three-dimensional environment, Hybrid Artificial Intelligence Systems, Lecture Notes in Computer Science, 6076 (Springer, Berlin Heidelberg: Springer, 2010), 24-31.

[12] D. Kurabayashi and K. Osagawa, Formation transition based on geometrical features for multiple autonomous mobile robots, Journal of the Robotics Society if Japan, 23(3), 2005, 104-110.

[13] H.Q. Min, J.H. Zhu, and X.J. Zheng, Obstacle avoidance with multi-objective optimization by PSO in dynamic environment, IEEE Proceedings of International Conference on Machine Learning and Cybernetics, 5, 2005, 2950-2956.

[14] RITA. Connected Vehicle Research in the United States, 2014, http://www.its.dot.gov/connected_vehicle/connected vehicle_research.htm, (accessed Aug. 28, 2015).

[15] F. Zhu and S.V. Ukkusuri, A linear programming formulation for autonomous intersection control within a dynamic traffic assignment and connected vehicle environment, Transportation Research Part C: Emerging Technologies, 55, 2015, 363-378.

[16] S.I. Guler, M. Menendez, and L. Meier, Using connected vehicle technology to improve the efficiency of intersections, Transportation Research Part C: Emerging Technologies, 46, 2014, 121-131.

[17] Y. Feng, K.L. Head, S. Khoshmagham, and M. Zamanipour, A real-time adaptive signal control in a connected vehicle environment, Transportation Research Part C: Emerging Technologies, $55,2015,460-473$.

[18] J.I. Ge and G. Orosz, Dynamics of connected vehicle systems with delayed acceleration feedback, Transportation Research Part C: Emerging Technologies, 46, 2014, 46-64. 
[19] B. Khondaker and L. Kattan, Variable speed limit: A microscopic analysis in a connected vehicle environment, Transportation Research Part C: Emerging Technologies, 58 (Part A), 2015, 146-159.

[20] L. Consolini and C.M. Verrelli, Learning control in spatial coordinates for the path-following of autonomous vehicles, Automatica, 50(7), 2014, 1867-1874.

[21] S. Yu and Z. Shi, Dynamics of connected cruise control systems considering velocity changes with memory feedback, Measurement, 64, 2015, 34-48.

[22] Z. Sarris, Survey of UAV applications in civil markets, $S T N$ ATLAS-3Sigma AE and Technical University of Crete, DPEM, Crete, Greece, 2001.

[23] A. Cesetti, E. Frontoni, A. Mancini, A. Ascani, P. Zingaretti, and S. Longhi, A visual global positioning system for unmanned aerial vehicles used in photogrammetric applications, Journal of Intelligent and Robotic Systems, 61, 2011, 157-168.

[24] G. Conte and P. Doherty, Vision-based unmanned aerial vehicle navigation using geo-referenced information, Journal of Advances in Signal Processing, 2009, 18, Volume 2009, Article ID 387308 .

[25] L. Babel, Flight path planning for unmanned aerial vehicles with landmark-based visual navigation, Robotics and Autonomous Systems, 62(2), 2014, 142-150.

[26] B.R. Donald, P.G. Xavier, J. Canny, and J. Reif, Kinodynamic planning, Journal of the ACM, 40, 1993, 1048-1066.

[27] S.M. LaValle, Planning Algorithms (New York: Cambridge University Press, 2006).

[28] J.C. Latombe, Robot Motion Planning (Boston, MA: Kluwer Academic Publishers, 1991).

[29] W.M. Carlyle, J.O. Royset, and R.K. Wood, Routing military aircraft with a constrained shortest-path algorithm, Military Operations Research, 14(3), 2009, 31-52.

[30] E. Rippel, A. Bar-Gill, and N. Shimkin, Fast graph-search algorithms for general aviation flight trajectory generation, Journal of Guidance, Control, and Dynamics, 28(4), 2005, $801-811$

[31] M. Zabarankin, S. Uryasev, and R. Murphey, Aircraft routing under the risk of detection, Naval Research Logistics, 53(8), 2006, 728-747.

[32] S.A. Bortoff, Path planning for UAVs, Proceedings of the American Control Conference, Chicago, IL, 2000, 364-368.

[33] K.B. Judd and T.W. McLain, Spline based path planning for unmanned air vehicles, AIAA Guidance, Navigation, and Control Conference and Exhibit, Montreal, Canada, 2000.

[34] C. Goerzen, Z. Kong, and B. Mettler, A survey of motion planning algorithms from the perspective of autonomous UAV guidance, Journal of Intelligent and Robotic Systems, 57, 2010, 65-100.

[35] Q. Deng, J. Yu, and N. Wang, Cooperative task assignment of multiple heterogeneous unmanned aerial vehicles using a modified genetic algorithm with multi-type genes, Chinese Journal of Aeronautics, 26(5), 2013, 1238-1250.

[36] H-Y. Kim, J-S. Lee, H-L. Choi, and J-H. Han, Autonomous formation flight of multiple flapping-wing flying vehicles using motion capture system, Aerospace Science and Technology, 39, 2014, 596-604.

\section{Biographies}

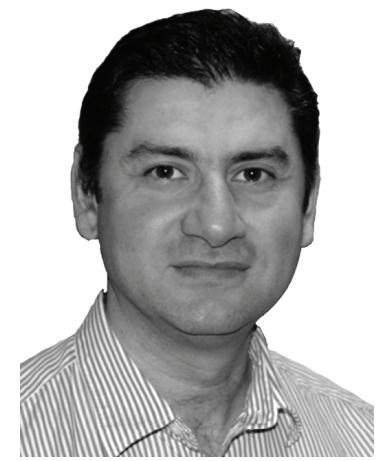

Farbod Khoshnoud is a Lecturer in the Department of Mechanical Engineering at Brunel University London, UK. His current research areas include Self-powered Dynamic Systems and Biologically Inspired Dynamic Systems. He was a lecturer at the University of Hertfordshire before joining Brunel, 2011-2014. He was a visiting scientist and postdoctoral researcher in the Industrial
Automation Laboratory, Department of Mechanical Engineering, at the University of British Columbia (UBC), Vancouver, 2007-2012. He was a visiting researcher at California Institute of Technology, 2009-2011. He carried out postdoctoral research in the Department of Civil Engineering at UBC, 2005-2007. He received his Ph.D. in Mechanical Engineering from Brunel University London in 2005. He has worked in industry as a mechanical engineer for over six years.

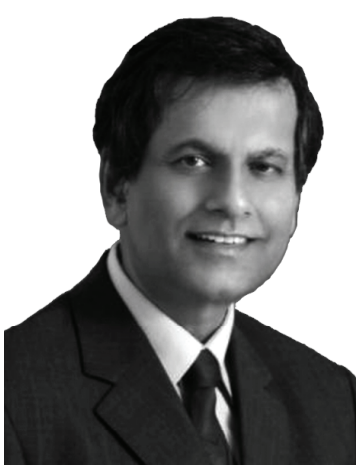

Clarence $W$. de Silva is a Fellow of IEEE, ASME, Canadian Academy of Engineering, and Royal Society of Canada. $\mathrm{He}$ received $\mathrm{Ph}$.D. degrees from Massachusetts Institute of Technology (1978; and University of Cambridge, UK (1998); and honorary D.Eng. degree from University of Waterloo, Canada (2008). He has been a Professor of Mechanical Engineering and NSERC-BC

Packers Chair in Industrial Automation, at the University of British Columbia, Vancouver, Canada, since 1988, and currently occupies the Senior Canada Research Chair in Mechatronics \& Industrial Automation. He has authored 22 books and about 475 papers, approximately half of which are in journals. His recent books published by Taylor \& Francis/CRC are Sensors and Actuators-Engineering System Instrumentation (2015); Mechanics of Materials (2014); Mechatronics-A Foundation Course (2010); Modeling and Control of Engineering Systems (2009); Modeling and Control of Engineering Systems (2009); VIBRATION-Fundamentals and Practice, 2nd Ed. (2007); and by Addison Wesley: Soft Computing and Intelligent Systems Design-Theory, Tools, and Applications (with F. Karray, 2004).

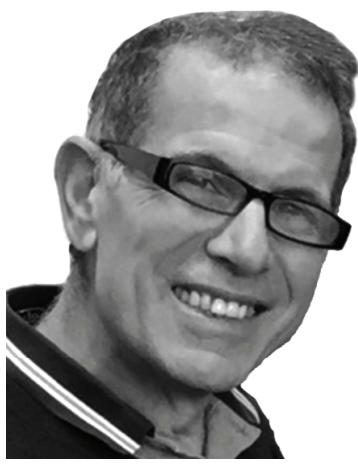

Ibrahim I. Esat graduated from Queen Mary College of London University with B.Sc. Honors and in the same university studied his Ph.D. degree in 1978 joining Newcastle University as a postdoctoral fellow. In 1980, he became the head of Mechanical Engineering Department of the newly formed Eastern Mediterranean University in Cyprus and two years later returned to the United Kingdom joining the University college of University of London as a research fellow. Following this in 1982, he joined the Dunlop Technology division as a Principle Engineer heading pioneering research in CAD/CAM developing in-house surface modeler and FEA for hyperelastic material. In 1984, he returned to Queen Mary College as a lecturer and since 1994, he has been at Brunel University, first as a reader and then as a full professor. Prof. Esat's research expertise covers areas such as modeling, design and 
optimization of mechanical systems, material characterization and modeling of materials, particularly rubber and viscoelastic materials and the use of biological inspired algorithms and computing in engineering problems. Over the past 20 years, he graduated more than 55 Ph.D. students and published more than 250 refereed technical papers. He is the sole author of a number of dynamic and vibration modeling software packages used in industry. He is currently the head of Applied Mechanics research group with over 20 staff and more than 30 researchers, and co-director of Brunel Orthopaedic Research and Learning Centre. His current research is in bioengineering, especially in musculoskeletal joint modeling, biologically inspired computing, and optimal vibration control. He is the recipient of many international awards from ASME, SDPS (Society of Design and Process Science), has given many invited talks, and chaired or co-chaired major international conferences. He was the president of the SDPS. 